\title{
Preventing cognitive decline in healthy older adults
}

\author{
Raza Naqvi MD, Dan Liberman MD, Jarred Rosenberg MD, Jillian Alston MD, Sharon Straus MD MSc
}

$\mathrm{G}$ iven the increase in the aging population, the prevention of cognitive decline in healthy older adults deserves close attention. Mild cognitive impairment affects 10\%-25\% of people over the age of 70 years. ${ }^{1}$ Mild cognitive impairment involves cognitive decline beyond that normally expected in a person of the same age with preservation of function. Dementia is defined as cognitive decline in one of several cognitive domains, along with difficulty in functional abilities. The annual rate of conversion from mild cognitive impairment to dementia is about $10 \%$. $^{2}$ Given this rate, in combination with the aging population, it is estimated that the prevalence of dementia will double to more than 1 million Canadians over the next 25 years. ${ }^{3}$

A summary of our search strategy is outlined in Box 1. Our search included randomized controlled trials (RCTs) involving participants with normal cognition. We also searched for RCTs involving participants with mild cognitive impairment. Given the substantial differences between people with normal cognition and those with mild cognitive impairment, this review focuses on people with normal baseline cognition, which constitutes most of the older population.

The prevention of cognitive decline is an important concern for many older adults. ${ }^{4}$ There are many organizations and industries that target the public with claims that use of their products will prevent cognitive decline. These products range from physical and cognitive programs to prescription and nonprescription medications. The evidence to support use of these products is sparse, and both the public and clinicians find it challenging to identify which strategies might be effective in preventing cognitive decline. Often, the data regarding these interventions are conflicting, and the studies are of poor quality. The objective of our review is to clarify the evidence with a comprehensive review of high-quality published studies that investigated strategies to prevent cognitive decline in healthy older adults, for the purpose of providing guidance to older adults and their clinicians. We defined cognitive decline as any decline found by neuropsychiatric testing or increase in the incidence of mild cognitive impairment or dementia.

\section{What pharmacologic interventions might be effective in preventing cognitive decline in older adults?}

There is no strong evidence for the use of any pharmacologic interventions to prevent cognitive decline in healthy older adults (Table 1). ${ }^{5-36}$ However, there is some evidence of harm with certain pharmacologic therapies, including estrogen therapies and anti-inflammatory drugs. ${ }^{8-11,28}$

\section{Cholinesterase inhibitors and $\mathrm{N}$-methyl-D-aspartate glutamate receptor antagonists}

Cholinesterase inhibitors and $N$-methyl-Daspartate (NMDA) receptor antagonists are approved for use in patients with dementia in an attempt to slow down cognitive and functional decline. Three RCTs investigated the effects of cholinesterase inhibitors or NMDA receptor antagonists for preventing cognitive decline in cognitively normal older adults..$^{5-7}$ Cholinesterase inhibitors prevent the breakdown of acetylcholine, a chemical involved in neurotransmission, by inhibiting acetylcholinesterase. The NMDA receptor antagonists are thought to selectively block the excitotoxic effects associated with abnormal transmission of glutamate. A total of 89 patients were included in these trials, and follow-up ranged from 3 to 15 months. One study investigating donepezil ${ }^{5}$ included 20 patients and showed improvement in immediate $(p=0.012)$ and delayed $(p=0.006)$ semantic recall at 10 -week follow-up, with no changes in immediate and delayed superficial recall. The
Competing interests: None declared.

Disclaimer: Sharon Straus is an associate editor for CMAJ. She was not involved in the editorial decision-making process for this article.

This article has been peer reviewed.

Correspondence to: Raza Naqvi, raza.naqvi@utoronto.ca

CMAJ 2013. DOI:10.1503 /cmaj.121448

\section{- Ker poInTs}

- There is no consistent evidence of benefit for any pharmacologic agent in preventing cognitive decline in healthy older adults.

- Studies investigating estrogen therapies and anti-inflammatory medications have shown evidence of a decline in memory.

- The evidence for physical activity in preventing cognitive decline is weak.

- Formal cognitive training exercises may have a benefit in preventing cognitive decline. 
2 other RCTs investigated donepezil and memantine, and showed no evidence of improved memory or cognition. ${ }^{6,7}$

\section{Hormonal therapies}

\section{Estrogen}

Seven RCTs investigated the effects of estrogen and its derivatives, including 4 trials coordinated by the Women's Health Initiative Memory Study (WHIMS) group..$^{8-14} \mathrm{~A}$ total of 10792 patients were involved in these RCTs, 10426 of whom were involved in the WHIMS trials. Follow-up of patients ranged from 4 to 5 years for the WHIMS trials $^{8-11}$ and from 2 weeks to 1 year for the other studies. ${ }^{12-14}$ These studies indicated a relative decline in cognitive function and an increase in incident dementia in patients in the treatment arms with hormonal therapy (hazard ratio 1.8, $95 \%$ confidence interval [CI] 1.2 to 2.6 ).

\section{Testosterone}

Three RCTs studied testosterone use in 144 men, with study follow-up ranging from 3 months to 3 years..$^{15-17}$ The results were conflicting, with the shortest study of 3 months showing some possible improvement in spatial $(p=0.01)$ and verbal $(p=0.05)$ memory. ${ }^{15}$ Another study indicated worsening in short-term memory $(p<0.05$, effect size 0.59) with testosterone treatment at 9 months, ${ }^{16}$ and the third study showed no significant change in memory-related outcomes over 3 years. ${ }^{17}$

\section{Dehydroepiandrosterone}

Dehydroepiandrosterone (DHEA) is a natural hormone that can be synthetically produced and is frequently marketed as an agent that can pre-

\section{Box 1: Evidence for this review}

- We searched MEDLINE, Embase, CINAHL (Cumulative Index to Nursing and Allied Health Literature) and Cochrane Central Register of Controlled Trials from the date of database onset until Oct. 31, 2011, using a list of search terms that included "cognitive decline," "dementia" and "mild cognitive impairment" (for the full list of terms, see Appendix 1, available at www.cmaj.ca/lookup/suppl/doi:10.1503/cmaj.121448/-/DC1). Our inclusion criteria were randomized controlled trials of pharmacologic or nonpharmacologic interventions in patients aged 65 years and older with normal baseline cognition or mild cognitive impairment. We included studies that investigated any form of cognitive decline, including the development of mild cognitive impairment, progression to dementia or worsening cognitive function on cognitive testing.

- The initial search found 5205 articles, and multiple levels of screening yielded 32 articles for the review (see Appendix 1 for screening algorithms). We assessed study quality using the criteria developed by the Cochrane Effective Practice and Organisation of Care group (http://epoc.cochrane.org /epoc-methods), which assesses risk of bias. Whereas the overall quality of the literature was moderate, most studies had substantial limitations in methodology or data analysis (see Appendix 2, available at www.cmaj.ca /lookup/suppl/doi:10.1503/cmaj.121448/-/DC1). vent cognitive decline. ${ }^{37}$ Three $\mathrm{RCTs} \mathrm{s}^{18-20}$ investigated the use of DHEA in a total of 317 patients. Follow-up was from 6 weeks to 1 year. None of the 3 studies showed a statistically significant improvement in cognitive function with the use of DHEA supplements.

\section{Ginkgo}

Ginkgo is a popular herbal supplement available worldwide that is thought to have antioxidant effects and to possibly affect neurotransmitters in a manner that is not well characterized..$^{38}$ Two RCTs have investigated the prevention of cognitive decline with ginkgo. ${ }^{21,22}$ One that followed 230 cognitively healthy older adults for 6 weeks did not show a significant difference in any cognitive outcome measured. ${ }^{21}$ Another RCT studied 118 patients over 42 months and also found no significant change in cognitive decline between the gingko and placebo groups. ${ }^{22}$

\section{Vitamins and fatty acids}

Randomized controlled trials have assessed the use of various vitamins and fatty acids for the prevention of cognitive decline. Vitamin $\mathrm{B}_{6}$ $\left(n=76\right.$, study duration $\left.12 \mathrm{wk}^{23}\right)$, vitamin $\mathrm{E}$ $\left(n=6377\right.$, study duration nearly $\left.10 \mathrm{yr}^{24}\right)$, folic acid $\left(n=24\right.$, study duration $\left.4 \mathrm{wk}^{25}\right)$ and the omega-3 fatty acid EPA-DHA (eicosapentaenoic acid-docosahexaenoic acid; $n=302$, study duration $6 \mathrm{mo}^{26}$ ) have all been studied, and none showed evidence of preventing cognitive decline.

\section{Miscellaneous pharmacologic interventions}

Four RCTs investigated other pharmacologic interventions to determine if they prevent cognitive decline in healthy older adults. ${ }^{27-30}$ One study examined the effects of candesartan over 4 years among nearly 5000 patients, but no significant change in cognition was noted with its use. ${ }^{27}$ A second study conducted over 3 years investigated the use of naproxen and celecoxib among 2500 patients and found a marginal decline in memory with use of the medications; global summary scores were 0.05 standard deviations lower $(p=0.02)$ in the treatment arm. ${ }^{28} \mathrm{~A} 4$-week study of a placebo versus no pill in 40 older adults reported an improvement in delayed recall with placebo ( $p=0.035$ ), but review of the CI for the point estimate showed that the result was not statistically significant (CI -0.3 to 1.3$).{ }^{29}$ Another study investigated AIT-082 $(n=9$, follow-up $5 \mathrm{wk}$ ), an oral nerve growth factor, which found no significant improvement in memory with intervention. ${ }^{30}$ 


\section{Do any nonpharmacologic interventions prevent cognitive decline?}

One RCT investigating resistance training in healthy older adults showed improvement in cognitive outcomes. ${ }^{31}$ There is consistent evidence that cognitive training using formal programs is effective at preventing cognitive decline based on 3 RCTs (Table 1). ${ }^{34-36}$

\section{Physical exercise}

Three RCTs have investigated the role of physical exercise in preventing cognitive decline. Cassilhas and colleagues ${ }^{31}$ investigated resistance-training protocols of moderate and high intensity compared with a placebo stretching group in a study involving 62 men over 6 months. The resistancetraining protocols consisted of 3 one-hour sessions per week beginning with a 10-minute warmup and then using varying resistance loads under professional supervision. The authors found a statistically significant improvement in some, but not all, tests of short-term and long-term episodic memory. ${ }^{31}$ The authors did not report an overall change in cognition.

A second RCT compared resistance- and balance-training exercises with a flexibility and relaxation program, as well as a no-exercise control. The study, which included 152 healthy older adults who were followed for 1 year, showed no significant improvement in visual, verbal or working memory with the intervention compared with the flexibility and relaxation program, or the no-exercise control..$^{32}$

Baker and colleagues ${ }^{33}$ completed a 6-month study with 28 participants comparing aerobic exercise (participants used treadmills, stationary bicycles or elliptical trainers to reach $75 \%-85 \%$ of their heart rate reserve) to stretching (participants carried out stretching and balancing exercises while maintaining their heart rates at or below $50 \%$ of their reserve). This study found benefits in executive function $(p=0.04)$ but not in memory in the aerobic exercise group. ${ }^{33}$

\section{Cognitive training}

Three RCTs explored the role of various forms of cognitive training, which can also be referred to as mental exercise, to prevent cognitive decline in healthy older adults. Willis and colleagues $^{34}$ investigated the role of cognitive training in reasoning, speed or memory in 2802 healthy older adults. All 3 groups showed significant improvements in memory over the 5-year follow-up period, with a relatively greater effect size in the memory-trained group compared with the reasoning- or speed-training groups (effect size $0.23 \mathrm{v}$. 0.05 ). The effect size was defined as the difference in improvement from baseline to year 5 between the training and control groups divided by intrasubject standard deviation of the Blom-transformed composite score.

Smith and colleagues ${ }^{35}$ compared a computerized cognitive training program based on brain plasticity with a general cognitive stimulation program over 8 weeks in 487 older adults. There was a significant improvement in auditory memory and attention in the treatment group compared with the control group $(p=0.02)$ with an improvement of 2.1 points (3.9 points, $95 \% \mathrm{CI}$ 2.7 to 5.1 , v. 1.8 points, $95 \%$ CI 0.6 to 3.0 ) on a 100-point scale $;^{35}$ however, the overlap in CIs

\begin{tabular}{|c|c|c|c|}
\hline Intervention & No. of patients & Study duration & $\begin{array}{l}\text { Overall effect } \\
\text { on memory }\end{array}$ \\
\hline $\begin{array}{l}\text { Cholinesterase inhibitors and NMDA } \\
\text { receptor antagonists } s^{5-7}\end{array}$ & 89 & 3-15 mo & None \\
\hline \multicolumn{4}{|l|}{ Hormonal therapies } \\
\hline Estrogen $^{8-14}$ & 10792 & $2 w k-5 y r$ & Worsened memory \\
\hline Testosterone $\mathrm{i}^{15-17}$ & 144 & $3 \mathrm{mo}-3 \mathrm{yr}$ & Inconsistent \\
\hline DHEA $^{18-20}$ & 317 & $6 \mathrm{wk}-1 \mathrm{yr}$ & Inconsistent \\
\hline Ginkgo $^{21,22}$ & 348 & $6 w k-3.5 y r$ & None \\
\hline Vitamins and fatty acids $s^{23-26}$ & 6779 & 4 wk-9.6 yr & None \\
\hline $\begin{array}{l}\text { Miscellaneous pharmacologic } \\
\text { interventions } s^{27-30}\end{array}$ & 7530 & $4 w k-3.7 y r$ & Inconsistent \\
\hline Physical exercise ${ }^{31-33}$ & 244 & $6 \mathrm{mo}-1 \mathrm{yr}$ & Inconsistent \\
\hline Cognitive training ${ }^{34-36}$ & 3321 & $3 w k-5 y r$ & Improvement \\
\hline
\end{tabular}


between the treatment and control groups indicates that the significance of the difference is unclear.

Berry and colleagues ${ }^{36}$ assessed the impact of 10 hours of computerized visual demonstration training compared with no training in a study of 32 people over 4 weeks. They found statistically significant improvement in performance after 4 weeks in the intervention group compared with the control group $(p<0.05$, effect size 0.81$)$, not only in the trained perceptual task but also in untrained working memory tasks. ${ }^{36}$

\section{Modification of vascular risk factors}

We found no RCTs that investigated the modification of vascular risk factors and their impact on the prevention of cognitive decline in healthy older adults. As mentioned, one RCT did look specifically at the use of candesartan and found no significant difference in cognitive outcomes over 4 years. ${ }^{27}$ However, we found no RCTs that investigated overall blood pressure control, weight reduction, smoking cessation or other interventions related to reduction of vascular risk factors that may be hypothesized to reduce cognitive decline.

\section{Limitations and future directions}

This review was limited to English-language studies. We did not include studies involving patients with mild cognitive impairment because this was outside the scope of our review. As with any review, we were limited by the quality of studies available in the literature. Notably, most studies had relatively short follow-up periods for interventions aimed at preventing cognitive decline. Furthermore, several studies selectively reported data and most used numerous end-

\section{Box 2: Applying the results of this review to clinical practice}

- When patients are seen in the primary care clinic and are interested in knowing whether they can do anything to prevent the onset of dementia or other forms of cognitive decline, clinicians can use the results of this review to inform the discussion.

- There is some evidence that specific cognitive exercises can sharpen memory and have an incremental benefit in preventing cognitive decline. However, the transferability of these benefits to any cognitively stimulating activity is unproven.

- There is some evidence that physical exercise may have a positive benefit, and given its countless other medical benefits, it should be encouraged with all patients.

- Perhaps the most important application of this review can be the rebuttal of claims of many natural health food products and other pharmacologic agents that purport benefits to cognition.

- For patients seeking further information and resources, clinicians may direct them to websites such as the following one created by the Alzheimer's Association: www.alz.org/we_can_help_brain_health _maintain_your_brain.asp. points, which made the significance of positive results questionable in the setting of many nonsignificant changes within the same study. The highly variable outcome measures in terms of various tests used to investigate memory outcomes makes it difficult to compare results across studies. One of the most substantial limitations in this review is that the changes observed do not appear to be clinically significant.

A recent Cochrane Review examined cognitively stimulating activities and their role in preventing cognitive decline in older adults with normal cognition or mild cognitive impairment. ${ }^{39}$ The study concluded that studies with no-activity controls showed significant improvement, whereas studies with active controls (compared with another intervention) did not reach statistical significance. ${ }^{39}$ Our review did not include studies with minimal cognitive impairment; however, one of the 3 studies that investigated cognitive training $^{35}$ did use an active control and found a relative benefit to a cognition-based training program.

This review provides some evidence to help clinicians and their patients address what strategies might prevent cognitive decline (Box 2). There is a lack of RCT data on other commonly proposed "anti-aging" strategies, including drinking wine and dietary restrictions, and thus we cannot comment on the potential benefit or harm of these strategies. Future studies should address the impact of cognitive training on the prevention of cognitive decline, and we encourage researchers to consider easily accessible tools such as crosswords puzzles and sudoku that have not been rigorously studied. The studies in this review that assessed cognitive exercises used exercises that were both labour- and resourceintensive, and thus may not be applicable to most patients.

\section{Conclusion}

We found 32 RCTs of interventions targeted to prevent cognitive decline in healthy older adults, but many were small and had short follow-up periods. None of the studies of pharmacologic agents found clinically or statistically significant benefits associated with their use. More promising results were seen in the studies that assessed cognitive training; all 3 RCTs showed some benefit in the prevention of cognitive decline (Box 2). However, the clinical significance of these results is not clear given that the changes on the cognitive scales used as outcomes in these studies were small. One of 3 studies assessing physical exercise, specifically resistance training, showed some potential benefit of physical training in preventing cognitive decline in healthy older adults. 
This review highlights that despite the importance of cognitive impairment, there is not a substantial body of literature addressing how it may be prevented.

\section{References}

1. Roberts RO, Geda YE, Knopman DS, et al. The Mayo Clinic Study of Aging: design and sampling, participation, baseline measures and sample characteristics. Neuroepidemiology 2008; 30:58-69.

2. Panza F, D'Introno A, Colacicco AM, et al. Current epidemiology of mild cognitive impairment and other predementia syndromes. Am J Geriatr Psychiatry 2005;13:633-44.

3. Alzheimer Society of Canada. Rising tide: the impact of dementia on Canadian society. Toronto (ON): The Society; 2010 Available: www.alzheimer.ca/en/Get-involved/Raise-your-voice /Rising-Tide/Rising-tide-summary (accessed 2013 Apr. 2).

4. Coley N, Ousset PJ, Andrieu S, et al. Memory complaints to the general practitioner: data from the GuidAge study. J Nutr Health Aging 2008; 12:66S-72S.

5. FitzGerald DB, Crucian GP, Mielke JB, et al. Effects of donepezil on verbal memory after semantic processing in healthy older adults. Cogn Behav Neurol 2008;21:57-64.

6. Ferris S, Schneider L, Farmer M, et al. A double-blind, placebocontrolled trial of memantine in age-associated memory impairment. Int J Geriatr Psychiatry 2007;22:448-55.

7. Jatoi A, Kahanic SP, Frytak S, et al. Donepezil and vitamin E for preventing cognitive dysfunction in small cell lung cancer patients: preliminary results and suggestions for future study designs. Support Care Cancer 2005;13:66-9.

8. Rapp SR, Espeland MA, Shumaker SA, et al. Effect of estrogen plus progestin on global cognitive function in postmenopausal women. JAMA 2003;289:2663-72.

9. Espeland MA, Rapp SR, Shumaker SA, et al. Conjugated equine estrogens and global cognitive function in postmenopausal women. JAMA 2004;291:2959-68.

10. Shumaker SA, Legault C, Rapp SR, et al. Estrogen plus progestin and the incidence of dementia and mild cognitive impairment in postmenopausal women. JAMA 2003;289:2651-62.

11. Shumaker SA, Legault C, Kuller L, et al. Conjugated equine estrogens and incidence of probable dementia and mild cognitive impairment in postmenopausal women. JAMA 2004;291:2947-58.

12. Wolf OT, Kudielka BM, Hellhammer DH, et al. Two weeks of transdermal estradiol treatment in postmenopausal elderly women and its effect on memory and mood: verbal memory changes are associated with the treatment induced estradiol levels. Psychoneuroendocrinology 1999;24:727-41.

13. Almeida OP, Lautenschlager NT, Vasikaran S, et al. A 20-week randomized controlled trial of estradiol replacement therapy for women aged 70 years and older: Effect on mood, cognition and quality of life. Neurobiol Aging 2006;27:141-9.

14. Jacobsen DE, Samson MM, Emmelot-Vonk MH, et al. Raloxifene improves verbal memory in late postmenopausal women: a randomized, double-blind, placebo-controlled trial. Menopause 2010;17:309-14.

15. Cherrier MM, Matsumoto AM, Amory JK, et al. The role of aromatization in testosterone supplementation: effects on cognition in older men. Neurology 2005;64:290-6.

16. Maki PM, Ernst M, London ED, et al. Intramuscular testosterone treatment in elderly men: evidence of memory decline and altered brain function. J Clin Endocrinol Metab 2007;92:4107-14.

17. Vaughan C, Goldstein FC, Tenover JL. Exogenous testosterone alone or with finasteride does not improve measurements of cognition in healthy older men with low serum testosterone. $J$ Androl 2007;28:875-82.

18. Wolf OT, Neumann O, Hellhammer DH, et al. Effects of a twoweek physiological DHEA substitution on cognitive performance and well-being in healthy elderly women and men. J Clin Endocrinol Metab 1997;82:2363-7.

19. van Niekerk JK, Huppert FA, Herbert J. Salivary cortisol and DHEA: association with measures of cognition and well-being in normal older men, and effects of three months of DHEA supplementation. Psychoneuroendocrinology 2001;26:591-612.

20. Kritz-Silverstein D, von Muhlen D, Laughlin GA, et al. Effects of DHEA supplementation on cognitive function and quality of life. J Am Geriatr Soc 2008;56:1292-8.

21. Solomon PR, Adams F, Silver A, et al. Ginkgo for memory enhancement. JAMA 2002;288:835-40.

22. Dodge HH, Zitzelberger T, Oken BS, et al. A randomized placebo-controlled trial of Ginkgo biloba for the prevention of cognitive decline. Neurology 2008;70:1809-17.

23. Deijen JB, van der Beek EJ, Orlebeke JF, et al. Vitamin B-6 supplementation in elderly men: effects on mood, memory, performance and mental effort. Psychopharmacology (Berl) 1992;109: 489-96.

24. Kang JH, Cook N, Manson J, et al. A randomized trial of vitamin E supplementation and cognitive function in women. Arch Intern Med 2006;166:2462-8.

25. Pathansali R, Mangoni AA, Creagh-Brown B, et al. Effects of folic acid supplementation on psychomotor performance and hemorheology in healthy elderly subjects. Arch Gerontol Geriatr 2006; 43:127-37.

26. van de Rest O, Geleijnse JM, Kok FJ, et al. Effect of fish oil on cognitive performance in older subjects. Neurology 2008;71:430-8.

27. Lithell H, Hansson L, Skoog I, et al. The study on cognition and prognosis in the elderly (SCOPE): principal results of a randomized double-blind intervention trial. J Hypertens 2003;21:875-86.

28. Martin BK, Szekely C, Brandt J, et al. Cognitive function over time in the Alzheimer's Disease Anti-Inflammatory Prevention Trial (ADAPT). Arch Neurol 2008;65:896-905.

29. Oken BS, Flegal K, Zajdel D, et al. Expectancy effect: impact of pill administration on cognitive performance in healthy seniors. J Clin Exp Neuropsychol 2008;30:7-17.

30. Grundman M, Farlow M, Peavy G, et al. A phase I study of AIT082 in healthy elderly volunteers. J Mol Neurosci 2002;18:283-93.

31. Cassilhas RC, Viana VA, Grassmann V, et al. The impact of resistance exercise on the cognitive function of the elderly. Med Sci Sports Exerc 2007;39:1401-7.

32. Brown AK, Liu-Ambrose T, Tate R, et al. The effect of groupbased exercise on cognitive performance and mood in seniors residing in intermediate care and self-care retirement facilities: randomised controlled trial. Br J Sports Med 2009;43:608-14.

33. Baker LD, Frank LL, Foster-Schubert K, et al. Aerobic exercise improves cognition for older adults with glucose intolerance, a risk factor for Alzheimer's disease. J Alzheimers Dis 2010;22:569-79.

34. Willis SL, Tennstedt SL, Marsiske M, et al. Long-term effects of cognitive training on everyday functional outcomes in older adults. JAMA 2006;296:2805-14.

35. Smith GE, Housen P, Yaffe K, et al. A cognitive training program based on principles of brain plasticity: results from the improvement in memory with plasticity-based adaptive cognitive training (IMPACT) study. J Am Geriatr Soc 2009;57:594-603.

36. Berry AS, Zanto TP, Clapp WC, et al. The influence of perceptual training on working memory in older adults. PLOS ONE 2010;5:e11537.

37. Stokel K. New research substantiates the anti-aging properties of DHEA. Life Extension Mag 2010 Dec. Available: www.lef.org /magazine/mag2010/dec2010_New-Research-Substantiates-the -Anti-Aging-Properties-of-DHEA_01.htm (accessed 2012 Aug. 23).

38. Christen Y. Oxidative stress and Alzheimer disease. Am J Clin Nutr 2000;71:621S-9S

39. Martin M, Clare L, Altgassen AM, et al. Cognition-based interventions for healthy older people and people with mild cognitive impairment. Cochrane Database Syst Rev 2011;(1):CD006220

Affiliations: From the Divisions of Geriatric Medicine (Naqvi, Liberman, Rosenberg, Straus) and General Internal Medicine (Alston), Department of Medicine, University of Toronto, Toronto, Ont.

Contributors: Raza Naqvi was involved in the literature search, abstract review, full-text review, data abstraction, manuscript drafting and editing. Dan Liberman was involved in the abstract review, full-text review, data abstraction and manuscript editing. Jarred Rosenberg and Jillian Alston were involved in the abstract review, data abstraction and manuscript editing. Sharon Straus was involved in the conception and design of the study and manuscript editing. All of the authors gave final approval of the version submitted for publication. 